\title{
Use of biotin targeted methotrexate-human serum albumin conjugated nanoparticles to enhance methotrexate antitumor efficacy
}

This article was published in the following Dove Press journal:

International Journal of Nanomedicine

7 September 201 I

Number of times this article has been viewed

\author{
Azade Taheri' \\ Rassoul Dinarvand ${ }^{1,2}$ \\ Faranak Salman Nouri' \\ Mohammad Reza \\ Khorramizadeh ${ }^{3}$ \\ Atefeh Taheri Borougeni ${ }^{4}$ \\ Pooria Mansoori ${ }^{5}$ \\ Fatemeh Atyabi ${ }^{1,2}$ \\ 'Department of Pharmaceutics, \\ Faculty of Pharmacy, Tehran University \\ of Medical Sciences, Tehran, Iran; \\ ${ }^{2}$ Nanotechnology Research Centre, \\ Faculty of Pharmacy, Tehran University \\ of Medical Sciences, Tehran, Iran; \\ ${ }^{3}$ Department of Pathobiology, School \\ of Public Health, Tehran University \\ of Medical Sciences, Tehran, Iran; \\ ${ }^{4}$ Department of Oral and Maxillofacial \\ Pathology, Faculty of Dentistry, Tehran \\ University of Medical Sciences, Tehran, \\ Iran; ${ }^{5}$ Faculty of Medicine, Ahvaz \\ Jundishapur University of Medical \\ Sciences, Ahvaz, Iran
}

\begin{abstract}
Biotin molecules could be used as suitable targeting moieties in targeted drug delivery systems against tumors. To develop a biotin targeted drug delivery system, we employed human serum albumin (HSA) as a carrier. Methotrexate (MTX) molecules were conjugated to HSA. MTX-HSA nanoparticles (MTX-HSA NPs) were prepared from these conjugates by cross-linking the HSA molecules. Biotin molecules were then conjugated on the surface of MTX-HSA NPs. The anticancer efficacy of biotin targeted MTX-HSA NPs was evaluated in mice bearing 4T1 breast carcinoma. A single dose of biotin targeted MTX-HSA NPs showed stronger in vivo antitumor activity than non-targeted MTX-HSA NPs and free MTX. By 7 days after treatment, average tumor volume in the biotin targeted MTX-HSA NPs-treated group decreased to $17.6 \%$ of the initial tumor volume when the number of attached biotin molecules on MTX-HSA-NPs was the highest. Average tumor volume in non-targeted MTX-HSA NPs-treated mice grew rapidly and reached $250.7 \%$ of the initial tumor volume. Biotin targeted MTX-HSA NPs increased the survival of tumor-bearing mice to $47.5 \pm 0.71$ days and increased their life span up to $216.7 \%$. Mice treated with biotin targeted MTX-HSA NPs showed slight body weight loss (8\%) 21 days after treatment, whereas non-targeted MTX-HSA NPs treatment at the same dose caused a body weight loss of $27.05 \% \pm 3.1 \%$.
\end{abstract}

Keywords: biotin, targeted drug delivery, in vivo anticancer delivery, human serum albumin, methotrexate, conjugates

\section{Introduction}

The side effects of anticancer drugs could limit the efficacy of cancer chemotherapy. ${ }^{1-2}$ Targeted drug delivery systems could diminish the adverse cytotoxic effects of anticancer drugs on normal tissues. ${ }^{3}$ Specific drug delivery to cancers could be achieved by adding a suitable targeting moiety on drug delivery systems. ${ }^{4}$ These targeting moieties could bind to certain types of receptors on the tumor cells and deliver cytotoxic drug to these cells specifically. Several targeting agents such as antibodies, ${ }^{5,6}$ peptides, ${ }^{7,8}$ vitamins, ${ }^{9}$ folic acid, ${ }^{10-14}$ and integrin ${ }^{15}$ could be used as suitable targeting moieties in targeted drug delivery systems. Among vitamins, biotin (vitamin H) has been used as a suitable targeting agent in several studies. ${ }^{16-18}$ Biotin receptors overexpressed on the surface of different cancer cells could internalize sufficiently the biotin targeted drug delivery system into these cells. ${ }^{18}$

In this study biotin was used as a targeting moiety to design a tumor targeted drug delivery system based on a human serum albumin (HSA) conjugation strategy. The biotin targeted drug delivery system consisted of (i) HSA as carrier; (ii) methotrexate (MTX) as anticancer drug; and (iii) biotin as a targeting moiety. MTX was conjugated
Correspondence: Rassoul Dinarvand Faculty of Pharmacy, Tehran University of Medical Sciences, I6th Azar St, Tehran |4|76|44II, Iran Tel +982166959095

$\mathrm{Fax}+982166959096$

Email dinarvand@tums.ac.ir 
to HSA and then MTX-HSA nanoparticles (MTX-HSA NPs) were prepared by cross-linking the HSA molecules using EDC cross-linker. The surface of MTX-HSA NPs was then functionalized by biotin molecules using a carbodiimide reaction. The targeting effect of biotin targeted MTX-HSA NPs to tumor cells were confirmed successfully in vitro. ${ }^{19}$

4T1 breast carcinoma derived from the Balb/c mammary carcinoma is a transplantable tumor cell line that is a highly malignant and invasive tumor model and can metastasize from the primary tumor in the mammary gland to other sites including lymph nodes, blood, liver, lung, brain, and bone. ${ }^{20}$ The 4T1 tumor resembles advanced breast cancer in humans and can be used as a suitable experimental model for human mammary cancer. ${ }^{21}$ The $4 \mathrm{~T} 1$ model can be used for determination of the effects of anticancer drugs such as MTX on breast tumor growth and metastasis efficiently. Moreover the 4T1 model can be used as a model for evaluation of biotin targeted drug delivery systems because of the overexpression of biotin receptors on the surface of these tumor cells. ${ }^{22}$ The aim of this study was to evaluate the antitumor effect of biotin targeted MTX-HSA NPs compared with non-targeted MTXHSA NPs on mice bearing 4T1 tumor in vivo. The effects of biotin targeted MTX-HSA NPs on the survival and body weight loss of 4T1 tumor-bearing mice were also evaluated.

\section{Materials and methods Materials}

Methotrexate USP was kindly donated by Cipla Pharmaceutical Co, India. Biotin was obtained from Sigma-Aldrich (St Louis, MO). 1-ethyl-3-(diaminopropyl) carbodiimde $\mathrm{HCl}$ (EDC), N-hydroxysuccinimide (NHS) and human serum albumin, HABA/Avidin and fluorescein isothiocyanate (FITC) were purchased from Sigma-Aldrich (Steinheim, Germany). RPMI-1640 modified medium and penicillin-streptomycin solution were obtained from Gibco Invitrogen (Calsbad, CA). Deionized water was used throughout the experiment. All other chemicals used were of reagent grade.

\section{Preparation of biotin functionalized MTX-HSA NPs}

MTX was conjugated to HSA by a carbodiimide reaction using EDC and NHS. ${ }^{23}$ Then MTX-HSA conjugates were cross-linked using EDC to form MTX-HSA NPs. For determination of MTX/HSA molar ratio in MTX-HSA NPs, free MTX was dissolved in $0.1 \mathrm{~N} \mathrm{NaOH}$. The absorbance of MTX solution was determined using UV spectrophotometry in $372 \mathrm{~nm}$. The calibration plot of MTX solution was linear in the range of $0.005-0.2 \mathrm{mg} / \mathrm{mL}$. It could be assumed that the molar absorptivity of MTX was not changed by conjugation to HSA. ${ }^{24}$ Thus, $12.5 \mathrm{mg}$ of MTX-HSA NPs was accurately weighed and redispersed in $4 \mathrm{~mL}$ of $0.1 \mathrm{~N} \mathrm{NaOH}$. MTX content was determined using UV absorbance of the MTXHSA NPs solution at $372 \mathrm{~nm}$. Moreover, HSA absorbance was negligible at this wavelength. HSA content of NPs was determined using a Total Protein Kit, Micro Lowry, Peterson's Modification (Sigma-Aldrich). In this study, we used MTXHSA NPs with an MTX/HSA molarity ratio of $8 \pm 0.18$. For conjugation of biotin on the surface of MTX-HSA NPs, $50 \mathrm{mg}$ of biotin, $25 \mathrm{mg}$ of NHS, and $40 \mathrm{mg}$ of EDC were dissolved in dimethyl sulfoxide (DMSO) (5 mL). The DMSO solution was placed at room temperature for 1 hour. Thirty milligrams of MTX-HSA NPs (MTX/HSA molar ratio = 8) were resuspended in $1 \mathrm{~mL}$ sodium bicarbonate solution $(0.1 \mathrm{M})$. The MTX-HSA NPs solution was divided into three equal parts. Different amounts of the DMSO solution (600, 1000 , and $1500 \mu \mathrm{L}$ ) were added to MTX-HSA NPs solutions. The obtained mixtures of MTX-HSA NPs and DMSO solution were stirred constantly at room temperature for 16 hours. After an appropriate time the mixture was dialyzed against phosphate-buffered saline (PBS) ( $\mathrm{pH}$ 7.2) using cellulose membrane (cutoff $12 \mathrm{kDa}$; Merck, Germany) overnight for removing unreacted biotin, N-hydroxysuccinimide, EDC, and DMSO. The biotin functionalized MTX-HSA NPs were lyophilized at $-40^{\circ} \mathrm{C}$ for 48 hours using a Lyotrap Plus lyophilizator (LTE, Scientific Ltd, Oldham, UK). The amount of biotin attached on the surface of MTX-HSA NPs was determined using HABA/Avidin complex. Biotin functionalized MTX-HSA NPs (1 mg) were resuspended in $1 \mathrm{~mL}$ of deionized water. HABA/Avidin solution $(900 \mu \mathrm{L}, 20 \mathrm{mg}$ in $1 \mathrm{~mL}$ deionized water) was added to $100 \mu \mathrm{L}$ of the solution containing biotin functionalized MTX-HSA NPs. Biotin content of the NPs was determined using UV spectrophotometry at $500 \mathrm{~nm}$ wavelength. ${ }^{25}$ The amount of biotin molecules was determined using the following formula:

$\Delta \mathrm{A} 500=0.9 \times(\mathrm{HABA} /$ Avidin absorbance $)$

- (HABA/Avidin and biotin functionalized MTX-HSA NPs absorbance)

$$
\text { Biotin concentration }(\mu \mathrm{M})=(\Delta \mathrm{A} 500 / 34) \times 10
$$

Three types of biotin functionalized MTX-HSA NPs with $3.66 \pm 0.22,7.01 \pm 0.14$ and $9.41 \pm 0.34$ biotin/HSA molar ratios were prepared. Biotin functionalized MTX-HSA NPs with $3.66 \pm 0.22,7.01 \pm 0.14$ and $9.41 \pm 0.34$ biotin/ HSA molar ratios were named biotin 3.66-MTX-HSA NPs, biotin 7.01-MTX-HSA NPs and biotin 9.41-MTX-HSA NPs, respectively. 


\section{Particle size and zeta potential measurement of biotin functionalized MTX-HSA NPs}

One milligram of the lyophilized biotin functionalized MTXHSA NPs was resuspended in $1 \mathrm{~mL}$ deionized water. Particle sizes, polydispersity, and zeta potential of the biotin functionalized MTX-HSA NPs were determined using Zetasizer ZS (Malvern Instruments, Malvern, UK).

Further characterization of NPs (eg, transmission electron microscopy images, fluorescence microscopy images of cellular uptake of NPs) and their in vitro drug release profile are given as supplementary data.

\section{Cell line}

Breast tumor 4T1 cell line was purchased from the American Type Culture Collection (Manassas, VA) and cultured in RPMI-1640 supplemented with $10 \%$ fetal bovine serum and $1 \%$ penicillin-streptomycin.

\section{Animal tumor model}

The ability of biotin-functionalized NPs to target MTX to tumors was evaluated in the 4T1 tumor model. The effect of single-dose biotin targeted MTX-HSA NPs, non-targeted MTX-HSA NPs, and free MTX treatment on tumor growth was investigated in mice bearing 4T1 tumor, which are known to overexpress biotin receptors..$^{22}$ All in vivo experiments were conducted according to the guidelines of ethical committee of the Pharmaceutical Research Centre, Faculty of Pharmacy, Tehran University of Medical Sciences, Iran. The female Balb/c mice of body weight 17-20 g were purchased from the Pasteur Institute (Tehran, Iran). 4T1 breast tumor cells $\left(10^{6}-10^{7}\right)$ were inoculated subcutaneously into the breast region of each female Balb/c mouse (6-8 weeks old) in a volume of $0.2 \mathrm{~mL}$. Animals had free access to food and water. When the tumor sizes of $95 \%$ of tumor-bearing mice were $>200 \pm 105 \mathrm{~mm}^{3}$, the mice were divided into 11 groups. There were 22 mice in the group treated with normal saline and seven mice in each of the other groups; five to six mice that had approximately the same tumor size were placed in one group. Two different doses of biotin targeted MTX-HSA NPs, non-targeted MTX-HSA NPs, and free MTX were injected to mice intravenously via the tail vein. The doses were equivalent to 6.25 and $12.5 \mathrm{mg} / \mathrm{kg}$ of free MTX. Normal saline was injected in to all animals of the control group. On day 0 before treatment, the mean tumor volumes of animals in groups treated with normal saline, free MTX $(6.25 \mathrm{mg} / \mathrm{kg})$, free MTX
(12.5 mg/kg), MTX-HSA NPs (equivalent MTX $6.25 \mathrm{mg} / \mathrm{kg}$ ), MTX-HSA NPs (equivalent MTX $12.5 \mathrm{mg} / \mathrm{kg}$ ), biotin 3.66-MTX-HSA NPs (equivalent MTX $6.25 \mathrm{mg} / \mathrm{kg}$ ), biotin 3.66-MTX-HSA NPs (equivalent MTX $12.5 \mathrm{mg} / \mathrm{kg}$ ), biotin 7.01-MTX-HSA NPs (equivalent MTX $6.25 \mathrm{mg} / \mathrm{kg}$ ), biotin 7.01-MTX-HSA NPs (equivalent MTX $12.5 \mathrm{mg} / \mathrm{kg}$ ), biotin 9.41-MTX-HSA NPs (equivalent MTX $6.25 \mathrm{mg} /$ $\mathrm{kg}$ ), and biotin 9.41-MTX-HSA NPs (equivalent MTX $12.5 \mathrm{mg} / \mathrm{kg}$ ) were $242.91 \pm 181.17,281.22 \pm 158.34$, $318.52 \pm 141.44,202.37 \pm 376,237.23 \pm 111.71$, $262.02 \pm 146.70,304.04 \pm 101.34,348.01 \pm 98.56$, $255.67 \pm 88.45,313.29 \pm 111.23,258.78 \pm 67.45$, respectively. The size of tumors at day 0 was taken as $100 \%$. Tumor sizes of each group were determined until 3 weeks after treatment with free MTX, biotin targeted MTX-HSA NPs, and non-targeted MTX-HSA NPs. The volume of tumors was measured using digital caliber and calculated using the following formula:

$$
0.4\left(\mathrm{~L} \cdot \mathrm{W}^{2}\right)
$$

$\mathrm{L}$ was the length and $\mathrm{W}$ was the width of the tumors. ${ }^{26}$

\section{Determination of median survival time and percentage increase in life span}

Animal survival time was monitored by recording the median survival time (MST) in days and percentage increase in life span (ILS\%) of mice treated with biotin targeted MTX-HSA NPs, non-targeted MTX-HSA NPs, and free MTX. Median survival time and percentage increase in lifespan (ILS\%) was calculated using the following formula: ${ }^{27}$

$$
\begin{aligned}
\text { Median survival time }(\text { MST })= & (\text { day of } 1 \text { st death } \\
& + \text { day of last death }) / 2
\end{aligned}
$$

Percentage increase

in life span $=[($ MST of treated group /MST of control group) -1$] \times 100$

\section{Evaluation of body weight loss}

The antitumor efficacy of biotin targeted MTX-HSA NPs could be evaluated by inhibitory effects of these NPs on the weight loss of tumor-bearing mice. ${ }^{28}$ Consequently the body weight of 4T1 tumor-bearing mice treated with free MTX, non-targeted and biotin targeted MTX-HSA NPs was also recorded every 3 days during the study. The doses were equivalent to $6.25 \mathrm{mg} / \mathrm{kg}$ of free MTX.

\section{Data analysis}

Data were analyzed by one-way analysis of variance (ANOVA). $P<0.05$ was considered statistically significant. 


\section{Results}

\section{Characterization of biotin functionalized MTX-HSA NPs}

The conjugation of biotin on the surface of MTX-HSA NPs (MTX/HSA molar ratio: 8) increased the size of these NPs from $111.7 \pm 4.6$ to $125-145 \mathrm{~nm}$ according to the amount of attached biotin molecules. The biotin functionalization of MTXHSA NPs increased the negative surface charge of these NPs from $-12.1 \pm 0.5 \mathrm{mV}$ to $-20.45 \pm 0.2 \mathrm{mV}$. Particle size, zeta potential, and biotin content for biotin functionalized and nonfunctionalized MTX-HSA NPs are summarized in Table 1. Further information regarding preparation and characterization of fractionalized MTX-HSA NPs are reported as supplements.

\section{In vivo anticancer efficacy of biotin targeted NPs}

As can be seen in Figure 1 tumor volumes in saline-treated mice increased rapidly (Figure 1). At the doses used (equivalent to 6.25 and $12.5 \mathrm{mg} / \mathrm{kg}$ free MTX), free MTX did not have a significant inhibitory effect on tumor growth compared with the control group (saline group). The inhibitory tumor growth effect of non-targeted MTX-HSA NPs was only 1.54to 1.98-fold more than free MTX at similar concentration. Conjugation of biotin molecules on the surface of MTXHSA NPs significantly enhanced inhibitory tumor growth effects of MTX-HSA NPs on the 4T1 tumor model. Biotin 7.01-MTX-HSA NPs at doses of 6.25 and $12.5 \mathrm{mg} / \mathrm{kg}$ of equivalent free MTX significantly $(P<0.05)$ reduced tumor volume in a dose-dependent manner compared with nontargeted NPs. A significant antitumor effect in mice treated with biotin targeted NPs was observed after 4 days and up to 21 days $(P<0.05)$ of treatment. At the end of the study of antitumor effect (day 21), mean tumor volume in the groups treated with biotin 9.41-MTX-HSA NPs, biotin 7.02-MTXHSA NPs, and biotin 3.66-MTX-HSA NPs was 8.46 $\pm 2.3-$, $4.88 \pm 1.23-$, and $3.74 \pm 1.05$-fold lower than that in the group treated with non-targeted MTX-HSA NPs (at doses equivalent to $12.5 \mathrm{mg} / \mathrm{kg}$ of free MTX) (Figure 2). By 7 days after injection, average tumor volume in groups treated with biotin 9.41-MTX-HSA NPs, biotin 7.02-MTX-HSA NPs, and biotin 3.66-MTX-HSA NPs $(12.5 \mathrm{mg} / \mathrm{kg}$ of equivalent free MTX) was only $17.67 \% \pm 7.78 \%, 28.56 \% \pm 8.46 \%$, and $34.44 \% \pm 8.22 \%$ of the initial tumor volume, whereas the average tumor volume in mice treated with non-targeted MTX-HSA NPs (equivalent to $12.5 \mathrm{mg} / \mathrm{kg}$ free MTX) grew rapidly and reached $250.78 \% \pm 29.01 \%$ of the initial tumor volume. Increasing the amount of attached biotin molecules on the surface of MTX-HSA NPs increased the antitumor effect of biotin targeted NPs (Figure 2).

\section{Biotin targeted NPs prolonged survival of tumor-bearing mice}

The effect of biotin targeted NPs on the survival of 4T1 tumor-bearing mice is shown in Figure 3 and Table 2. Biotin targeting increased the median survival time of 4T1 tumorbearing mice significantly compared with non-targeted MTX-HSA NPs. MST of the control group that received only saline was $15.01 \pm 0.79$ days, whereas it was $17.51 \pm 0.60$, $22.52 \pm 0.5,32.5 \pm 0.41,37.5 \pm 0.67$, and $47.5 \pm 0.71$ days for groups treated with free MTX, non-targeted MTX-HSA NPs, biotin 3.66-MTX-HSA NPs, biotin 7.02-MTX-HSA, NPs, and biotin 9.41-MTX-HSA NPs, respectively (equivalent to $6.25 \mathrm{mg} / \mathrm{kg}$ free MTX). The increase in the life span of 4T1 tumor-bearing mice treated with free MTX and non-targeted MTX-HSA NPs was $16.66 \%$ and $50.10 \%$, respectively. The increase in the life span of mice treated with biotin 3.66-MTXHSA NPs, biotin 7.02-MTX-HSA NPs, and biotin 9.41-MTXHSA NPs was $116.66 \%, 150.1 \%$, and $216.66 \%$, respectively.

\section{Evaluation of body weight loss}

There were no significant differences in weight change among the groups treated with biotin 3.66-MTX-HSA NPs,

Table I Physico-chemical characteristics of MTX-HSA NPs (MTX/HSA molar ratio $=8$ ) and biotin functionalized MTX-HSA NPs (mean \pm standard deviation; $\mathrm{n}=3$ )

\begin{tabular}{|c|c|c|c|c|}
\hline \multirow[t]{2}{*}{ Biotin/HSA molar ratio } & $\begin{array}{l}\text { MTX-HSA } \\
\text { NPs }\end{array}$ & $\begin{array}{l}\text { Biotin 3.66-MTX-HSA } \\
\text { NPs }\end{array}$ & $\begin{array}{l}\text { Biotin 7.0I-MTX-HSA } \\
\text { NPs }\end{array}$ & $\begin{array}{l}\text { Biotin 9.4 I-MTX-HSA } \\
\text { NPs }\end{array}$ \\
\hline & 0 & $3.66 \pm 0.22$ & $7.01 \pm 0.14$ & $9.41 \pm 0.34$ \\
\hline Particle size (nm) & $111.70 \pm 4.60$ & $125.10 \pm 3.40$ & $132.40 \pm 2.14$ & $144.36 \pm 3.80$ \\
\hline Poly dispersity index & $0.10 \pm 0.01$ & $0.21 \pm 0.07$ & $0.19 \pm 0.04$ & $0.24 \pm 0.05$ \\
\hline Zeta potential (mV) & $-12.10 \pm 0.5$ & $-20.23 \pm 1.11$ & $-19.56 \pm 2.01$ & $-21.54 \pm 1.25$ \\
\hline
\end{tabular}

Abbreviations: MTX-HSA NPs, methotrexate-human serum albumin conjugated nanoparticles; biotin 3.66-MTX-HSA NPs, biotin targeted methotrexate-human serum albumin nanoparticles: $3.66 \pm 0.22$ biotin/human serum albumin molar ratio; biotin 7.01-MTX-HSA NPs, biotin targeted methotrexate-human serum albumin nanoparticles: $7.01 \pm 0.14$ biotin/human serum albumin molar ratio; biotin 9.41 -MTX-HSA NPs, biotin targeted methotrexate-human serum albumin nanoparticles: $9.41 \pm 0.34$ biotin/ human serum albumin molar ratio. 


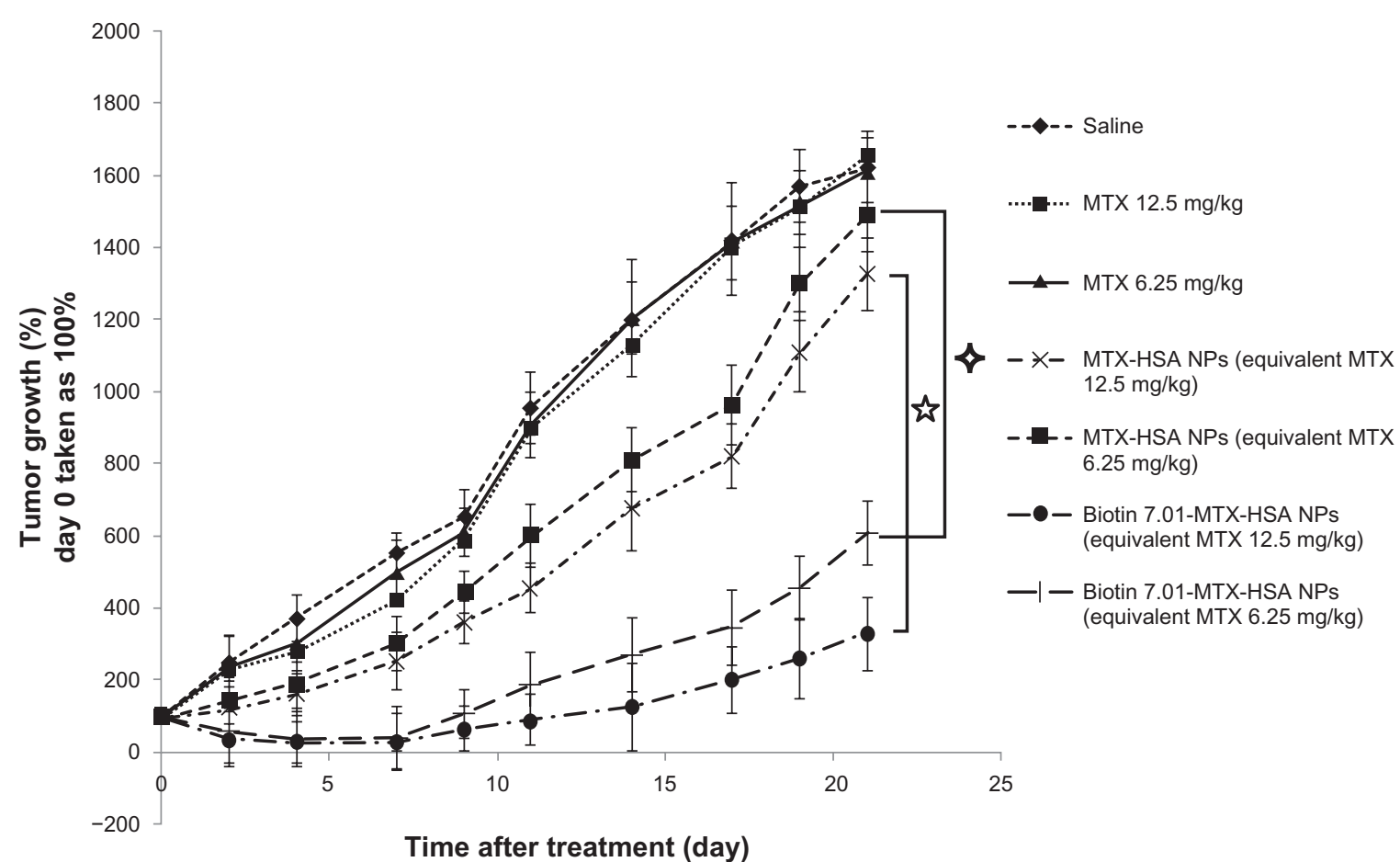

Figure I Antitumor effect of free MTX, MTX-HSA NPs, and biotin 7.0I-MTX-HSA NPs on 4TI tumor-bearing mice. 4TI tumor cells were implanted subcutaneously in Balb/c mice. The drugs were injected intravenously in a single dose (day 0). The doses were equivalent to 6.25 and $12.5 \mathrm{mg} / \mathrm{kg}$ of free MTX. Data are presented as mean \pm standard deviation of relative tumor volumes (day 0 taken as $100 \%$ ).

Abbreviations: MTX, methotrexate; MTX-HSA NP, methotrexate-human serum albumin conjugated nanoparticle; biotin 7.01-MTX-HSA NPs, biotin targeted methotrexate-human serum albumin nanoparticles: $7.01 \pm 0.14$ biotin/human serum albumin molar ratio.

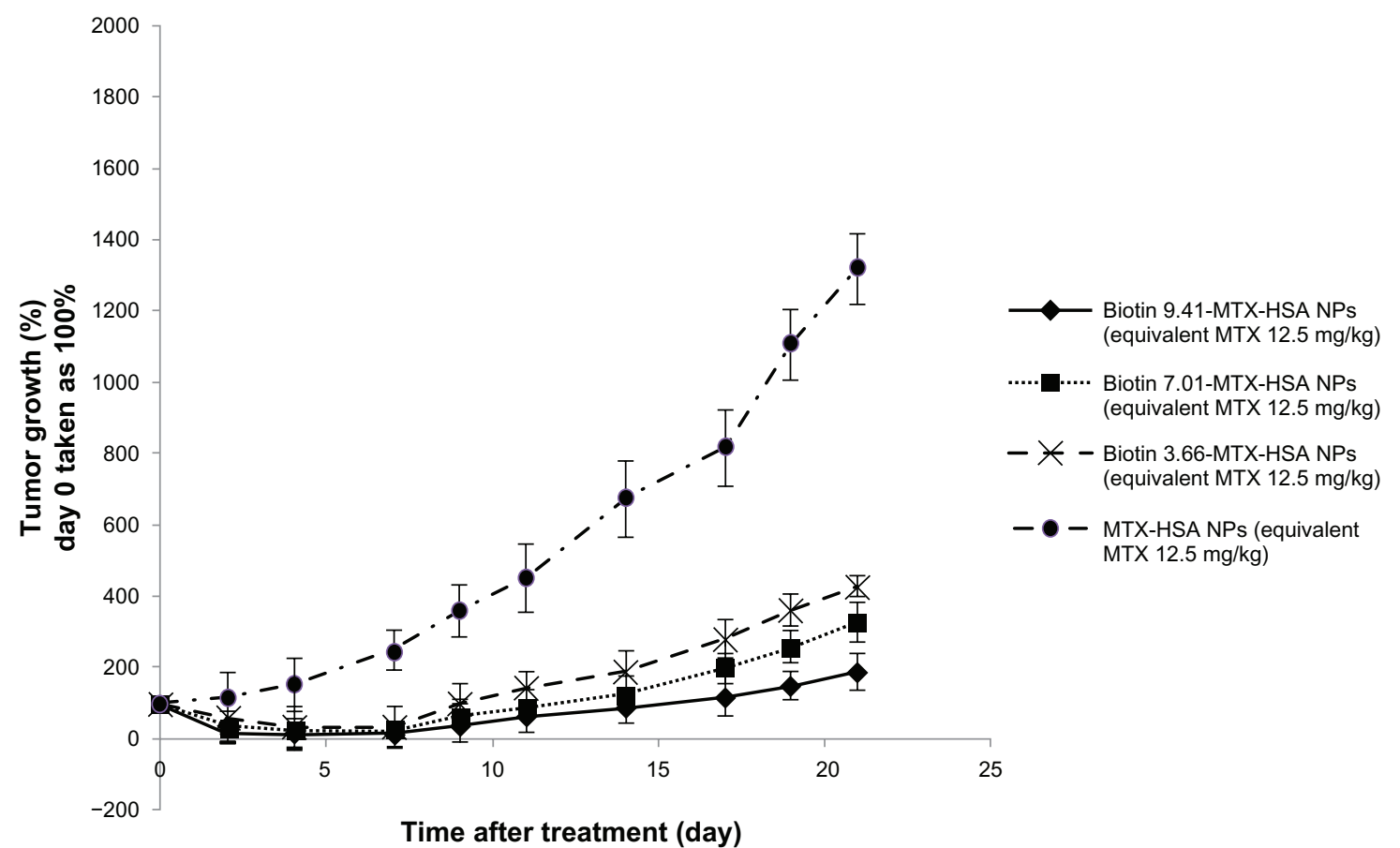

Figure 2 Antitumor effect of MTX-HSA NPs, biotin 3.66-MTX-HSA NPs, biotin 7.0I-MTX-HSA NPs, and biotin 9.4I-MTX-HSA NPs on 4TI tumor-bearing mice. 4TI tumor cells were implanted subcutaneously in Balb/c mice. The drugs were injected intravenously in a single dose (day 0 ). The doses were equivalent to I2.5 mg/kg of free MTX. Data are presented as mean \pm standard deviation of relative tumor volumes (day 0 taken as $100 \%$ ).

Abbreviations: MTX, methotrexate; MTX-HSA NP, methotrexate-human serum albumin conjugated nanoparticle; biotin 3.66-MTX-HSA NPs, biotin targeted methotrexate-human serum albumin nanoparticles: $3.66 \pm 0.22$ biotin/human serum albumin molar ratio; biotin 7.0I-MTX-HSA NPs, biotin targeted methotrexate-human serum albumin nanoparticles: $7.01 \pm 0.14$ biotin/human serum albumin molar ratio; biotin 9.4I-MTX-HSA NPs, biotin targeted methotrexate-human serum albumin nanoparticles: $9.41 \pm 0.34$ biotin/human serum albumin molar ratio. 


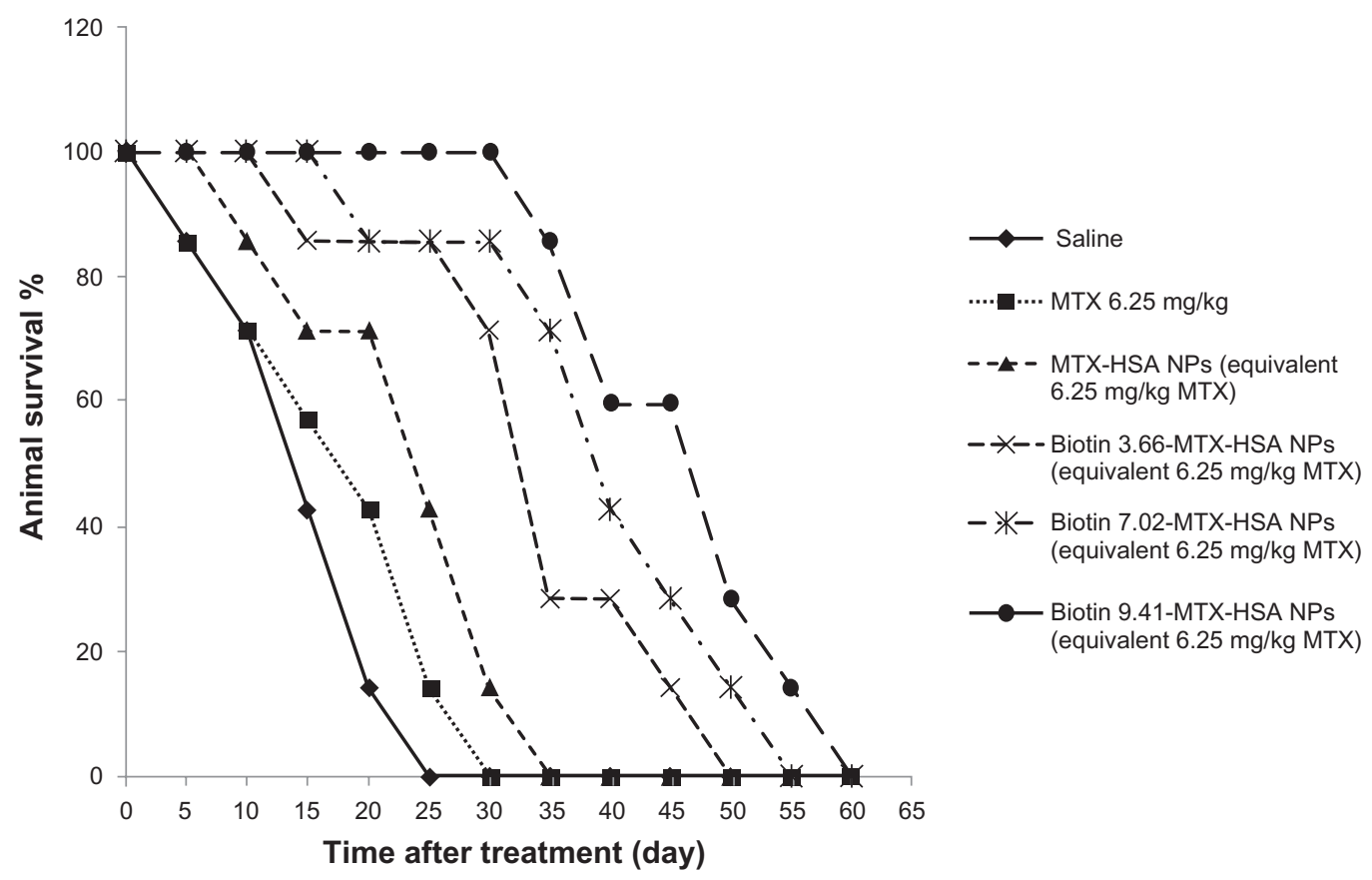

Figure 3 Animal survival study. The 4TI tumor-bearing mice were treated with free MTX, MTX-HSA NPs, biotin 3.66-MTX-HSA NPs, biotin 7.02-MTX-HSA NPs, and biotin 9.4I-MTX-HSA NPs. The drugs were injected intravenously in a single dose (day 0 ). The doses were equivalent to I $2.5 \mathrm{mg} / \mathrm{kg}$ of free MTX. The curve shows the number of 4TI tumor-bearing mice still alive on different days.

Abbreviations: MTX, methotrexate; MTX-HSA NP, methotrexate-human serum albumin conjugated nanoparticle; biotin 3.66-MTX-HSA NPs, biotin targeted methotrexate-human serum albumin nanoparticles: $3.66 \pm 0.22$ biotin/human serum albumin molar ratio; biotin 7.02-MTX-HSA NPs, biotin targeted methotrexate-human serum albumin nanoparticles: $7.01 \pm 0.14$ biotin/human serum albumin molar ratios; biotin $9.4 \mathrm{I}-\mathrm{MTX}-\mathrm{HSA}$ NPs, biotin targeted methotrexate-human serum albumin nanoparticles: $9.41 \pm 0.34$ biotin/human serum albumin molar ratio.

biotin 7.02-MTX-HSA NPs, and biotin 9.41-MTX-HSA NPs (Figure 4), nor were there significant differences between groups treated with free MTX and non-targeted MTX-HSA NPs and the group that received only saline. But the weight change of the groups treated with biotin targeted MTX-HSA NPs differed significantly from that of the group treated with non-targeted MTX-HSA NPs, especially between days 9 and 21 . A body weight loss of $<8 \%$ of initial body weight would be almost tolerable. ${ }^{28}$ The body weight loss of tumor-

Table 2 Effect of biotin targeting on the survival of 4TI tumorbearing mice

\begin{tabular}{lcl}
\hline Group & $\begin{array}{l}\text { Median survival } \\
\text { time (day) }\end{array}$ & $\begin{array}{l}\text { Life span } \\
\text { increase (\%) }\end{array}$ \\
\hline Saline & $15.01 \pm 0.79$ & - \\
MTX & $17.51 \pm 0.60$ & 16.66 \\
MTX-HSA NPs & $22.52 \pm 0.50$ & 50.10 \\
Biotin 3.66-MTX-HSA NPs & $32.5 \pm 0.41$ & 116.66 \\
Biotin 7.0I-MTX-HSA NPs & $37.5 \pm 0.67$ & 150.10 \\
Biotin 9.4I-MTX-HSA NPs & $47.5 \pm 0.71$ & 216.66 \\
\hline
\end{tabular}

Note: *The dose was equivalent to $6.25 \mathrm{mg} / \mathrm{kg}$ of free MTX.

Abbreviations: MTX, methotrexate; MTX-HSA NPs, methotrexate-human serum albumin conjugated nanoparticles; biotin 3.66-MTX-HSA NPs, biotin targeted methotrexate-human serum albumin nanoparticles: $3.66 \pm 0.22$ biotin/human serum albumin molar ratios; biotin 7.02-MTX-HSA NPs, biotin targeted methotrexatehuman serum albumin nanoparticles: $7.01 \pm 0.14$ biotin/human serum albumin molar ratio; biotin 9.4I-MTX-HSA NPs, biotin targeted methotrexate-human serum albumin nanoparticles: $9.41 \pm 0.34$ biotin/human serum albumin molar ratio. bearing mice treated with biotin targeted MTX-HSA NPs was $<8 \%$ of initial body weight, but mice treated with free MTX and non-targeted MTX-HSA NPs lost $21.9 \% \pm 5.1 \%$ and $18.7 \% \pm 6.4 \%$ of their body weight, respectively, after 12 days.

\section{Discussion}

The use of HSA NPs in targeted drug delivery systems has been well reported. ${ }^{29,30}$ HSA represents a biodegradable drug carrier system with the capacity to deliver a large payload of cytotoxic drug to the tumor site..$^{31,32}$ The free amino and carboxylic acid groups of HSA could be used for covalent coupling of cytotoxic drugs and targeting moieties. ${ }^{31,33-35}$ Therefore HSA NPs have been proposed as a suitable drug carrier system for targeted drug delivery to specific tumor sites. ${ }^{36}$ Conjugation of cytotoxic drugs to HSA could improve their antitumor efficacy and decrease their toxic side effects. ${ }^{32}$ Because of the overexpression of biotin receptors on the surface of many tumor cells, biotin molecules could be used as a suitable targeting moiety in targeted drug delivery systems. Exploring this approach, Patil et a ${ }^{17}$ recently have reported the specific targeting of MCF-7 and 4T1 tumor cells with biotin molecules coupled on the PEG-PLA NPs containing paclitaxel and tariquidar (a P-gp modulator). They showed 


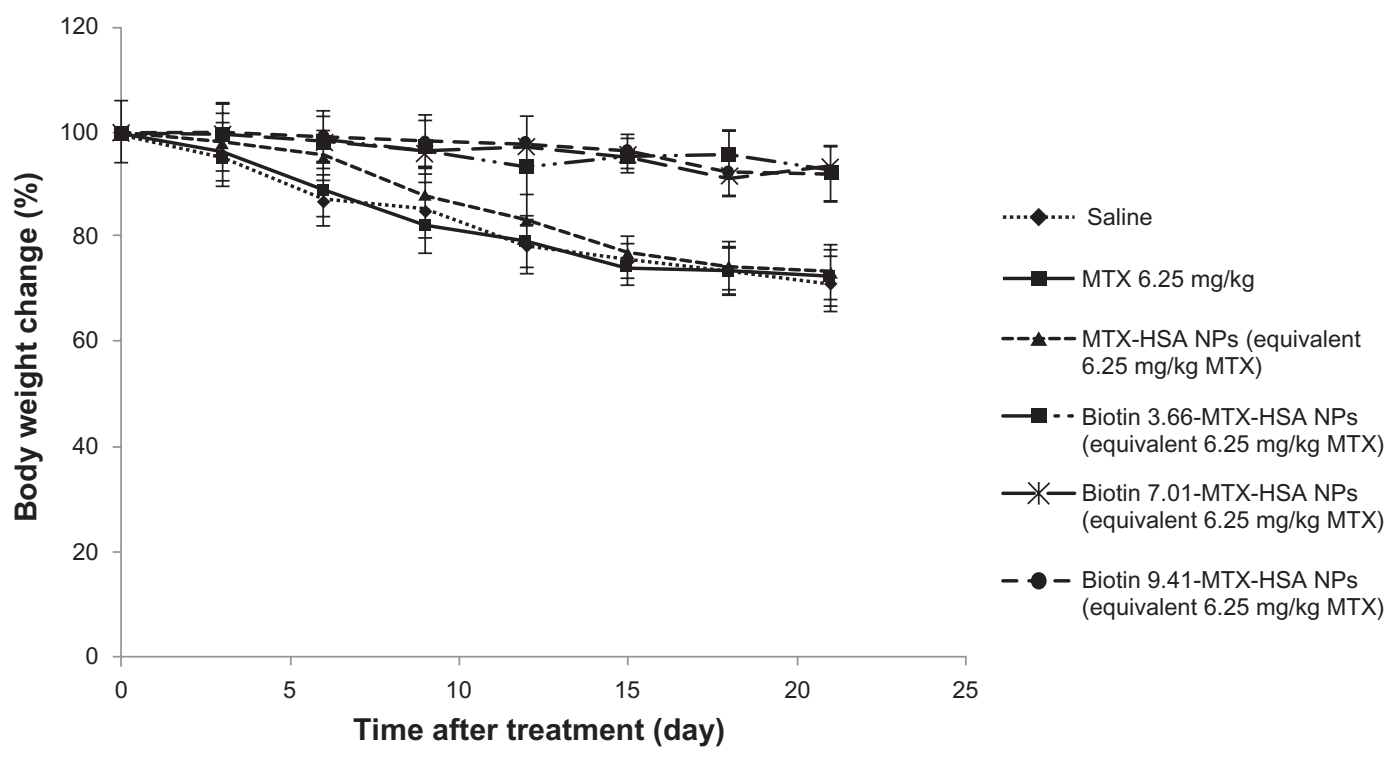

Figure 4 Change in body weight of 4TI tumor-bearing mice treated with free MTX, MTX-HSA NPs, biotin 3.66-MTX-HSA NPs, biotin 7.0I-MTX-HSA NPs, and biotin 9.4I-MTX-HSA NPs. The drugs were injected intravenously in a single dose (day 0 ). The doses were equivalent to $6.25 \mathrm{mg} / \mathrm{kg}$ of free MTX.

Abbreviations: MTX, methotrexate; MTX-HSA NPs, methotrexate-human serum albumin conjugated nanoparticles; biotin 3.66-MTX-HSA NPs, biotin targeted methotrexate-human serum albumin nanoparticles: $3.66 \pm 0.22$ biotin/human serum albumin molar ratio; biotin 7.0I-MTX-HSA NPs, biotin targeted methotrexate-human serum albumin nanoparticles: $7.01 \pm 0.14$ biotin/human serum albumin molar ratios; biotin 9.4I-MTX-HSA NPs, biotin targeted methotrexate-human serum albumin nanoparticles: $9.41 \pm 0.34$ biotin/human serum albumin molar ratios.

that the uptake of biotin functionalized PEG-PLA NPs by tumor cells in vitro was specific. Moreover, biotin targeted paclitaxel loaded NPs had much higher antitumor effects than non-targeted NPs in vivo.

In our previous study, we first developed MTX-HSA conjugates, and then MTX-HSA conjugated NPs by cross-linking of HSA molecules using EDC cross-linker. ${ }^{23}$ MTX-HSA NPs showed suitable cytotoxic effects in vitro but their delivery to the tumor site is through passive targeting. Active targeting, mediated by specific receptors on the surface of tumor cells, could increase the uptake of targeted NPs by tumor cells. To increase the antitumor effect of MTX-HSA NPs, we functionalized these NPs by biotin molecules. ${ }^{19}$ Three types of biotin functionalized MTX-HSA NPs with biotin/HSA molar ratios of 3.66, 7.01, and 9.41 were prepared. After biotin conjugation, the size of MTX-HSA NPs was increased slightly in proportion to the amount of attached biotin molecules. The zeta potential of these NPs was decreased to $-20 \mathrm{mV}$ when functionalized with biotin molecules. The decrease in zeta potential may be attributed to the decrease of the free amino groups on the MTX-HSA NPs after conjugation of the biotin molecules to these groups. The results of a previous study showed that the cytotoxic effects of biotin targeted MTX-HSA NPs on T47D and HeLa tumor cells increased significantly compared with non-targeted MTX-HSA NPs and free MTX in vitro. ${ }^{19}$ Moreover, decreasing the cellular uptake of biotin targeted MTX-HSA NPs in the presence of free biotin molecules confirmed the specific biotin receptormediated endocytosis of biotin targeted NPs. Fluorescence microscopy images also confirmed the much higher uptake of biotin targeted MTX-HSA NPs in T47D and HeLa tumor cells compared with non-targeted MTX-HSA NPs. ${ }^{19}$ In the present study, the antitumor efficacy of biotin targeted MTXHSA NPs on 4T1 tumor-bearing mice was investigated, and the results reinforced the results of in vitro studies. The results of in vivo study showed that free MTX alone could not inhibit tumor growth sufficiently and there were no significant differences between the tumor size of the group treated with free MTX and the control group. The inhibitory effect of non-targeted MTX-HSA NPs on tumor growth was slightly better than that of free MTX in 4T1 tumor-bearing mice. But tumor growth in groups treated with biotin targeted MTXHSA NPs was significantly slower than in those treated with non-targeted MTX-HSA NPs. Tumor volume 21 days after treatment in mice treated with biotin 9.41-MTX-HSA NPs, biotin 7.02-MTX-HSA NPs, and biotin 3.66-MTX-HSA NPs (equivalent to $12.5 \mathrm{mg} / \mathrm{kg}$ free MTX) was, respectively, $1134.11 \% \pm 89 \%, 994.10 \% \pm 34 \%$, and $899.02 \% \pm 48 \%$ less than that of those treated with non-targeted MTX-HSA NPs. Similar to in vitro results, biotin targeted MTX-HSA NPs with more attached biotin molecules on their surfaces had more in vivo antitumor effects compared to NPs with less biotin molecules. Moreover, biotin targeted MTX-HSA NPs prolonged survival time of $4 \mathrm{~T} 1$ tumor-bearing mice compared 
with non-targeted MTX-HSA NPs. Prolonged survival time of groups treated with biotin targeted MTX-HSA NPs could be related to potent inhibitory effects of tumor growth of these NPs, as evidenced by antitumor efficacy results. Lower body weight loss in groups treated with biotin targeted MTXHSA NPs might be related to the better antitumor effects or lower toxicity (side effects) of biotin targeted NPs compared with non-targeted MTX-HSA NPs and free MTX. MTX has various side effects such as weakness, tiredness, and body weight loss. MTX may also disturb food uptake and consequently cause body weight loss. ${ }^{37}$ It is possible that biotin targeting decreased the uptake of MTX-HSA NPs in normal organs and decreased the side effects of MTX in these organs. Consequently, biotin targeting could transport MTX to the tumor site with great efficiency and increase its antitumor effect sufficiently in tumor-bearing mice.

\section{Conclusion}

Biotin targeted human serum albumin-anticancer drug conjugated NPs were developed as a suitable targeted drug delivery system to enhance therapeutic effects of MTX and reduce its side effects. Biotin targeted MTX-HSA NPs could accumulate in cells with overexpression of biotin receptors. The in vivo anticancer experiment showed that biotin targeted MTX-HSA NPs had stronger antitumor activity and lower toxic effect than non-targeted MTX-HSA NPs and free MTX in a mouse breast tumor model. Therefore, biotin targeted MTX-HSA NPs could be used as a potent anticancer drug for treatment of tumors.

\section{Disclosure}

The authors declare no conflicts of interest in relation to this paper.

\section{References}

1. Fennelly D. Dose intensity in advanced ovarian cancer: have we answered the question? Clin Cancer Res. 1995;1(6):575-582.

2. Kobayashi M, Wood PA, Hrushesky WJ. Circadian chemotherapy for gynecological and genitourinary cancers. Chronobiol Int. 2002;19(1):237-251.

3. Minko T, Dharap SS, Pakunlu RI, Wang Y. Molecular targeting of drug delivery systems to cancer. Curr Drug Targets. 2004;5(4):389-406.

4. Dinarvand R, Sepehri N, Manoochehri S, Rouhani H, Atyabi F. Polylactide-co-glycolide nanoparticles for controlled delivery of anticancer agents Int J Nanomedicine. 2011;6:877-895.

5. Cho N, Chueh PJ, Kim C, Caldwell S, Morre DM, Morre D J. Monoclonal antibody to a cancer-specific and drug-responsive hydroquinone (NADH) oxidase from the sera of cancer patients. Cancer Immunol Immunother. 2002;51(3):121-129.

6. Lukyanov AN, Elbayoumi TA, Chakilam AR, Torchilin VP. Tumortargeted liposomes: doxorubicin-loaded long-circulating liposomes modified with anti-cancer antibody. J Control Release. 2004;100(1): 135-144.
7. Dharap SS, Wang Y, Chandna P, et al. Tumor-specific targeting of an anticancer drug delivery system by LHRH peptide. PANS. 2005;102(36):12962-12967.

8. He Y, Zhang L, Song C. Luteinizing hormone-releasing hormone receptor-mediated delivery of mitoxantrone using LHRH analogs modified with PEGylated liposomes. Int J nanomedicine. 2010;5:697-705.

9. Chen S, Zhao X, Chen J, et al. Mechanism-based tumor-targeting drug delivery system validation of efficient vitamin receptor-mediated endocytosis and drug release. Bioconjug Chem. 2010;21(5):979-987.

10. Zhao D, Zhao X, Zu Y, et al. Preparation, characterization, and in vitro targeted delivery of folate-decorated paclitaxel-loaded bovine serum albumin nanoparticles. Int J Nanomedicine. 2010;5:669-677.

11. Saltan N, Kutlu MH, Hür D, Iscan A, Say R. Interaction of cancer cells with magnetic nanoparticles modified by methacrylamido-folic acid. Int J Nanomedicine. 2011;6:477-484.

12. Swanson SD, Kukowska-Latallo JF, Patri AK, et al. Targeted gadoliniumloaded dendrimer nanoparticles for tumor-specific magnetic resonance contrast enhancement. Int J Banomedicine. 2008;3(2):201-210.

13. Li Q, Liu C, Zhao X, et al. Preparation, characterization and targeting of micronized 10-hydroxycamptothecin-loaded folate-conjugated human serum albumin nanoparticles to cancer cells. Int J Nanomedicine. 2011;6:397-405.

14. Li X, Tian X, Zhang J, et al. In vitro and in vivo evaluation of folate receptor-targeting amphiphilic copolymer-modified liposomes loaded with docetaxel. Int J Nanomedicine. 2011;6:1167-1184.

15. Xie J, Shen Z, Li KCP, Danthi N. Tumor angiogenic endothelial cell targeting by a novel integrin-targeted nanoparticle. Int J Nanomedicine. 2007;2(3):479-485.

16. Yang W, Cheng Y, Xu T, Wang X, Wen L-p. Targeting cancer cells with biotin dendrimer conjugates. Eur J Med Chem. 2009;44(2):862-868.

17. PatilY, Sadhukha T, Ma L, Panyam J. Nanoparticle-mediated simultaneous and targeted delivery of paclitaxel and tariquidar overcomes tumor drug resistance. J Control Release. 2009;136(1):21-29.

18. Na K. Self-assembled nanoparticles of hydrophobically-modified polysaccharide bearing vitamin $\mathrm{H}$ as a targeted anti-cancer drug delivery system. Eur J Pharm Sci. 2003;18(2):165-173.

19. Taheri A, Dinarvand R, Atyabi F, et al. Targeted delivery of methotrexate to tumor cells by means of biotin functionalized methotrexate-human serum albumin nanoparticles. J Biomed Nanotechnol. 2011. In press.

20. Tao K, Alroy J, Sahagian GG. Imagable 4 T1 model for the study of late stage breast cancer. BMC Cancer. 2008;8:228.

21. Aslakson CJ, Miller FR. Selective events in the metastatic process defined by analysis of the sequential dissemination of subpopulations of a mouse mammary tumor. Cancer Res. 1992;152:1399-1405.

22. Russell-jones, Mcewan J, inventors; Access Pharmaceuticals Australia PTY LTD, assignee. Amplification of biotin-mediated targeting. United States patent US 20060127310. June 15, 2006.

23. Taheri A, Atyabi F, Salman Nouri F, et al. Nanoparticles of conjugated methotrexate-human serum albumin: Preparation and cytotoxicity evaluations. J Nanomater. 2011:Article ID 768201.

24. Kosasih A, Bowman BJ, Wigent RJ, Ofner III CM. Characterization and in vitro release of methotrexate from gelatin/methotrexate conjugates formed using different preparation variables. Int J Pharm. 2000;204(1-2):81-89.

25. Green NC. A spectrophotometric assay for avidin and biotin based on binding of dyes by avidin. Biochem J. 1965;94:23C-24C.

26. Vredenburg MR, Ojima I, Veith J, et al. Effects of orally active taxanes on P-glycoprotein modulation and colon and breast carcinoma drug resistance. J Natl Cancer Inst. 2001;93(16):1234-1245.

27. Geran RI, Greenberg NH, Mac Donald MM, Schumacher AM, Abbot BJ. Protocols for screening chemical agents and natural products against animal tumors and other biological systems. Cancer Chemo Rep. 1972;3:1-103.

28. Tseng C, Su W, Yen K, Yang K, Lin F. The use of biotinylatedEGF-modified gelatin nanoparticle carrier to enhance cisplatin accumulation in cancerous lungs via inhalation. Biomaterials. 2009;30(20):3476-3485. 
29. Anhorn MG, Wagner S, Kreuter J, Langer K, Briesen H. Specific targeting of HER 2 overexpressing breast cancer cells with doxorubicinloaded trastuzumab-modified human serum albumin nanoparticles. Bioconjugate Chem. 2008;19(12):2321-2333.

30. Wagner S, Rothweiler F, Anhorn MG, et al. Enhanced drug targeting by attachment of an anti av integrin antibody to doxorubicin loaded human serum albumin nanoparticles. Biomaterials. 2010;31(8):2388-2398 .

31. Zhang L, Hou S, Mao S, Wei D, Song X, Lu Y. Uptake of folateconjugated albumin nanoparticles to the SKOV3 cells. Int J Pharm. 2004;287(1-2):155-162.

32. Kratz F. Albumin as a drug carrier: Design of prodrugs, drug conjugates and nanoparticles. J Control Release. 2008;132(3):171-183.

33. Kreuter J, Hekmatara T, Dreis S, Vogel T, Gelperina S, Langer K. Covalent attachment of apolipoprotein A-I and apolipoprotein B-100 to albumin nanoparticles enables drug transport into the brain. J Control Release. 2007;118(1):54-58.
34. Steinhauser I, Spankuchb B, Strebhardt K, Langer K. Trastuzumabmodified nanoparticles: Optimisation of preparation and uptake in cancer cells. Biomaterials. 2006;27(28):4975-4983.

35. Esmaeili F, Dinarvand R, Ghahremani MH, et al. Docetaxel-albumin conjugates: Preparation, in vitro evaluation and biodistribution studies. J Pharm Sci. 2009;98(8):2718-2730.

36. Tuan V, Chuang G, Kragh-Hansen U, Otagiri M. Pharmaceutical strategies utilizing recombinant human serum albumin. Pharm Res. 2002;19(5):569-577.

37. de Koning BAE, van Dieren JM, Lindenbergh-Kortleve DJ, et al. Contributions of mucosal immune cells to methotrexate-induced mucositis. Int Immunol. 2006;18(6):941-949. 


\section{Supplementary data}

The biotin targeted MTX-HSA NPs were characterized in vitro as below:

\section{Methods}

\section{Transmission electron microscopy}

The morphology of the biotin functionalized MTX-HSA NPs was studied by transmission electron microscopy (TEM). A drop of the biotin functionalized MTX-HSA NPs in water was placed on a graphite surface and was analyzed by TEM (Zeiss EM 900, Jena, Germany) at a voltage of $8 \mathrm{kV}$.

\section{In vitro release of MTX from biotin}

\section{functionalized NPs}

The release of free MTX from biotin functionalized MTXHSA NPs was evaluated in PBS pH 7.4 and serum. Twenty milligrams of biotin 3.6-MTX-HSA NPs, biotin 7.0-MTXHSA NPs and biotin 9.4-MTX-HSA NPs were redispersed separately in $2 \mathrm{~mL}$ of PBS $\mathrm{pH}$ 7.4. The solutions were introduced in to a dialysis tube (cellulose membrane, cutoff 12,000 Da, Merck, Germany). The tubes were placed into capped, wide-mouth jars containing $10 \mathrm{~mL}$ of PBS pH 7.4 as release medium. These jars were placed in a shaker water bath $\left(37^{\circ} \mathrm{C}\right)$ for 72 hours. The amount of free MTX was determined in $0.1 \mathrm{~mL}$ of release medium every 24 hours using high-performance liquid chromatography (HPLC) on a Teknokroma C18, $4.6 \times 250 \mathrm{~mm}$ column using a mobile phase consisting of phosphate buffer $\mathrm{pH}$ 7.2:acetonitrile in the volume ratio of 88:12 (flow rate of $1.20 \mathrm{~mL} / \mathrm{min}$ ) at $305 \mathrm{~nm}$ (UV detector, WellChrom, K-2600, Knauer, Germany).

\section{Stability of biotin functionalized MTX-HSA NPs}

To determine the stability of biotin functionalized MTX-HSA NPs, these NPs were resuspended in PBS pH 7.4 (25 mg in $4 \mathrm{~mL}$ ) and incubated at $4^{\circ} \mathrm{C}$ for 6 weeks. The size and zeta potential of NPs were measured every week. Every week $100 \mu \mathrm{L}$ of nanoparticle suspension was removed and diluted by deionized water to $1 \mathrm{~mL}$. The diluted suspension of NPs were centrifuged using Amicon ${ }^{\circledR}$ Ultra-4 Centrifugal Filter Devices (cutoff: $30 \mathrm{kDa}$ ) (Millipore, USA). The filtrate was evaluated for the amount of free MTX by HPLC on a Teknokroma C18, $4.6 \times 250 \mathrm{~mm}$ column using a mobile phase consisting of phosphate buffer $\mathrm{pH}$ 7.2:acetonitrile in the volume ratio of $88: 12$ (flow rate of $1.20 \mathrm{~mL} / \mathrm{min}$ ) at $305 \mathrm{~nm}$ (UV detector, WellChrom, K-2600, Knauer, Germany). Moreover the amount of free biotin in filtrate was analyzed using HABA/Avidin.

\section{Fluorescence microscopy}

To obtain direct evidence of the internalization of biotin functionalized MTX-HSA NPs compared with non-functionalized MTX-HSA NPs, biotin functionalized MTX-HSA NPs and non-functionalized MTX-HSA NPs were labeled by FITC. T47D and HeLa cells were seeded in 6-well plates (Costar, IL at a density of 300,000 cells per well for 24 hours. After 24 hours, T47D and HeLa cells were incubated with fresh culture medium containing FITC-labeled biotin functionalized MTX-HSA NPs and FITC-labeled non-functionalized MTX-HSA NPs at a concentration of $1 \mathrm{mg} / \mathrm{mL}$ for 4 hours at $37^{\circ} \mathrm{C}$. After 4 hours, cells were washed 3 times with PBS and the internalization of NPs to cells was visualized using fluorescence microscopy (Olympus IX 71, Japan).

\section{Results}

\section{TEM image}

TEM image of biotin functionalized MTX-HSA NPs (Figure S1) reveals their homogeneous size distribution.

\section{In vitro release of MTX from biotin functionalized NPs}

After 72 hours incubation of biotin functionalized MTX-HSA NPs in PBS pH 7.4 only $4.22 \% \pm 0.21 \%, 5.10 \% \pm 0.34 \%$, and $4.56 \% \pm 0.55 \%$ of MTX were released from biotin 3.6-MTX-HSA NPs, biotin 7.0-MTX-HSA NPs, and biotin

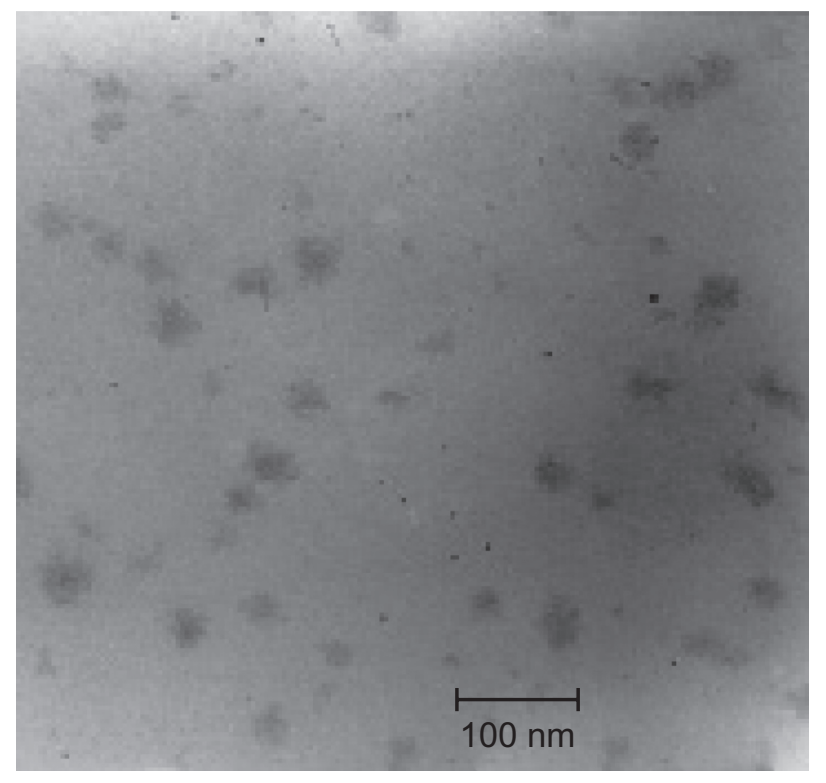

Figure SI Transmission electron microscopy image of biotin 7.0I-MTX-HSA NPs. Abbreviations: MTX-HSA NP, methotrexate-human serum albumin conjugated nanoparticle; biotin 7.01-MTX-HSA NPs, biotin targeted methotrexate-human serum albumin nanoparticles: $7.01 \pm 0.14$ biotin/human serum albumin molar ratio. 
9.4-MTX-HSA NPs, respectively. After incubation of biotin targeted MTX-HSA NPs in serum, $9.35 \% \pm 0.45 \%$, $10.46 \% \pm 0.12 \%$ and $10.11 \% \pm 0.33 \%$ of MTX were released.

\section{Stability studies}

The release of free MTX and biotin from biotin functionalized MTX-HSA NPs was estimated in aqueous buffered solution at $4{ }^{\circ} \mathrm{C}$. After storage, at least $96.2 \%$ of MTX was still linked to HSA and only $1.2 \%$ of biotin molecules had been released. The size and zeta potential of these NPs remained unchanged after 6 weeks at $4{ }^{\circ} \mathrm{C}$ (Figure S2).

\section{Fluorescence microscopy}

Internalization of FITC-labeled biotin functionalized MTXHSA NPs and FITC-labeled non-functionalized MTX-HSA NPs by T47D and HeLa cells was also investigated by

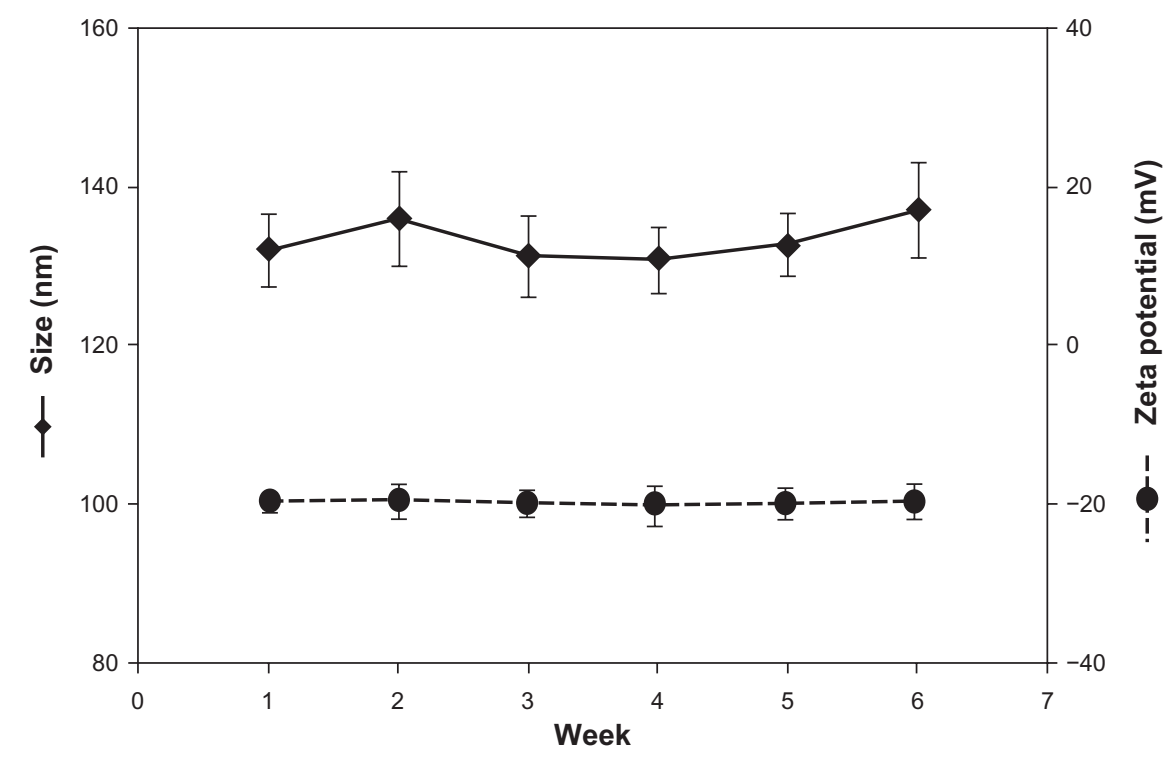

Figure S2 Storage stability of biotin 7.0I-MTX-HSA NPs. Size and zeta potential of NPs were recorded for 6 weeks.

Abbreviations: NP, nanoparticle; MTX-HSA NP, methotrexate-human serum albumin conjugated nanoparticle; biotin 7.0I-MTX-HSA NPs, biotin targeted methotrexatehuman serum albumin nanoparticles: $7.01 \pm 0.14$ biotin/human serum albumin molar ratio.
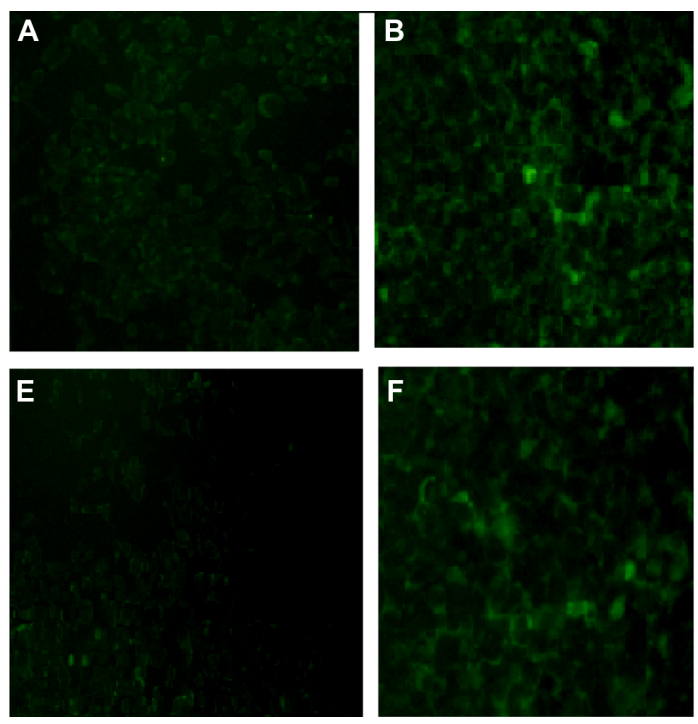
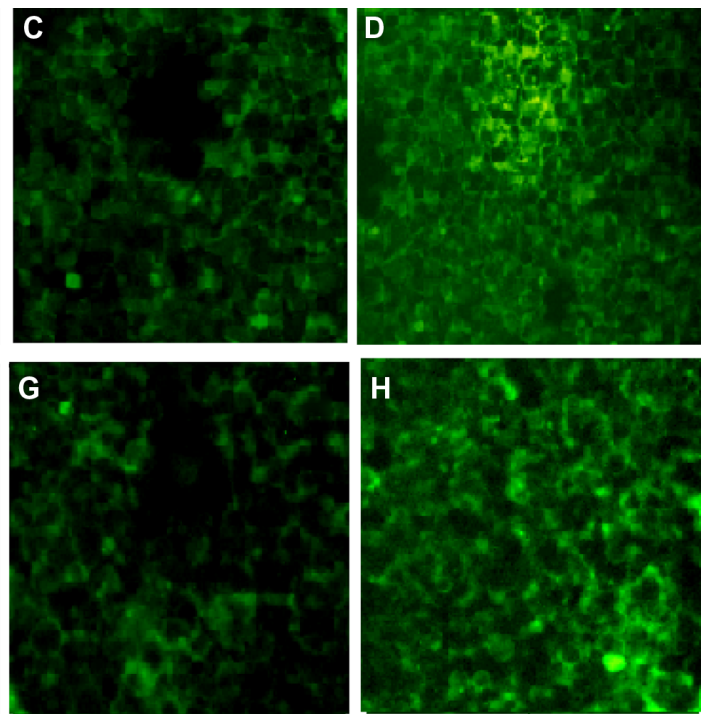

Figure S3 The uptake of FITC-labeled non-targeted MTX-HSA NPs (A) biotin 3.66-MTX-HSA NPs (B), biotin 7.0I-MTX-HSA NPs (C), biotin 9.4I-MTX-HSA NPs (D) at concentration of $\mathrm{I} \mathrm{mg} / \mathrm{mL}$ to T47D tumor cells after 4 hours at $37^{\circ} \mathrm{C}$. The uptake of FITC-labeled non-targeted MTX-HSA NPs (E), biotin 3.66-MTX-HSA NPs (F), biotin 7.0I-MTX-HSA NPs (G), biotin 9.4I-MTX-HSA NPs (h) at concentration of I mg/mL to HeLa tumor cells after 4 hours at $37^{\circ} \mathrm{C}$.

Abbreviations: FITC, fluorescein isothiocyanate; MTX, methotrexate; MTX-HSA NP, methotrexate-human serum albumin conjugated nanoparticle; biotin 3.66-MTX-HSA NPs, biotin targeted methotrexate-human serum albumin nanoparticles: $3.66 \pm 0.22$ biotin/human serum albumin molar ratio; biotin $7.0 \mathrm{I}-\mathrm{MTX}-\mathrm{HSA}$ NPs, biotin targeted methotrexate-human serum albumin nanoparticles: $7.0 \mathrm{I} \pm 0.14$ biotin/human serum albumin molar ratio; biotin 9.4I-MTX-HSA NPs, biotin targeted methotrexate-human serum albumin nanoparticles: $9.4 \mathrm{I} \pm 0.34$ biotin/human serum albumin molar ratio. 
fluorescence microscopy. After 4 hours incubation of T47D cells with the FITC-labeled biotin functionalized MTXHSA NPs and FITC labeled non-functionalized MTX-HSA NPs at $37^{\circ} \mathrm{C}$, T47D cells treated with FITC-labeled biotin functionalized MTX-HSA NPs (Figure S3B-D) showed significantly more intense fluorescence than T47D cells treated with FITC-labeled non-functionalized MTX-HSA NPs (Figure S3A). The uptake of FITC-labeled biotin functionalized MTX-HSA NPs in HeLa cells (Figure S3F-H) was also greater than that of FITC-labeled non-functionalized MTX-HSA NPs (Figure S3E).

\section{Publish your work in this journal}

The International Journal of Nanomedicine is an international, peerreviewed journal focusing on the application of nanotechnology in diagnostics, therapeutics, and drug delivery systems throughout the biomedical field. This journal is indexed on PubMed Central, MedLine, CAS, SciSearch ${ }^{\circledR}$, Current Contents ${ }^{\circledR} /$ Clinical Medicine,
Journal Citation Reports/Science Edition, EMBase, Scopus and the Elsevier Bibliographic databases. The manuscript management system is completely online and includes a very quick and fair peer-review system, which is all easy to use. Visit http://www.dovepress.com/ testimonials.php to read real quotes from published authors. 